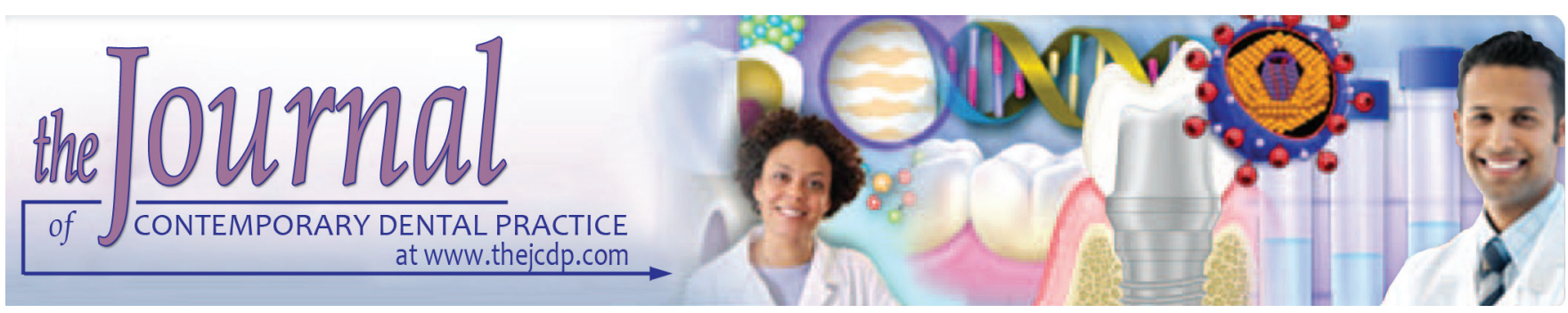

\title{
Comparison of Proinflammatory Cytokine Levels in Gingival Crevicular Fluid around Dental Implants with Ceramic and Titanium Abutments
}

${ }^{1}$ Ramin Negahdari, ${ }^{2}$ Mahdi Rahbar, ${ }^{3}$ Vahid Fakhrzadeh, ${ }^{4}$ Hosein Eslami, ${ }^{5}$ Taleb Akbari, ${ }^{6}$ Sepideh Bohluli

\begin{abstract}
Background: Considering the high success rate of osseointegration, there is ever-increasing use of dental implants. The mechanisms and biologic response of peri-implant tissues are different depending on the biocompatibility of the implant material. The aim of this study was to compare the proinflammatory cytokine levels in the gingival crevicular fluid (GCF) around dental implants with ceramic and titanium abutments.
\end{abstract}

Materials and methods: All the patients with dental implants referring to two private offices of two prosthodontists from June to August 2016 were examined in relation to implant health, and eligible subjects, based on inclusion criteria, were included in this study with a slit-mouth design. In this context, on one side titanium implants and, on the other side, ceramic implants were used. Samples were collected from the peri-implant sulcus in each patient and sent to the immunology laboratory for determination of interleukin (IL)-6 and IL-1 $\beta$ proinflammatory cytokine levels using an enzyme-linked immunosorbent assay kit. Data were analyzed with Statistical Package for the Social Sciences version 16. Statistical significance was set at $p<0.05$.

Results: The results showed lower levels of IL-1 $\beta$ and IL- 6 in the GCF around ceramic abutments compared with titanium implants. In addition, statistical comparison between IL-6 and IL-1 $\beta$ levels showed higher levels of IL- 6 around titanium and ceramic abutments compared with IL-1 $\beta$ levels.

\footnotetext{
${ }^{1,3}$ Department of Prosthodontics, Faculty of Dentistry, Tabriz University of Medical Sciences, Tabriz, Iran

${ }^{2}$ Department of Operative and Esthetic Dentistry, Faculty of Dentistry, Tabriz University of Medical Science, Tabriz, Iran

${ }^{4,6}$ Department of Oral Medicine, Faculty of Dentistry, Tabriz University of Medical Sciences, Tabriz, Iran

${ }^{5}$ Private Practitioner, Tabriz, Iran

Corresponding Author: Sepideh Bohluli, Department of Oral Medicine, Faculty of Dentistry, Tabriz University of Medical Sciences, Tabriz, Iran, Phone: +04133355965, e-mail: sepid. bohlouli@gmail.com
}

Conclusion: Based on the results of this study, the human body immune response to ceramic abutments is much better than that to titanium implants.

Clinical significance: Considering that proinflammatory cytokine levels in GCF around ceramic abutments are lower than titanium ones, ceramic abutments are preferred for clinical usage.

Keywords: Ceramic abutment, Cytokine, Dental implant, Gingival crevicular fluid, Titanium abutment.

How to cite this article: Negahdari R, Rahbar M, Fakhrzadeh V, Eslami H, Akbari T, Bohluli S. Comparison of Proinflammatory Cytokine Levels in Gingival Crevicular Fluid around Dental Implants with Ceramic and Titanium Abutments. J Contemp Dent Pract 2017;18(9):831-836.

Source of support: Nil

Conflict of interest: None

\section{INTRODUCTION}

At present, there are ever-increasing efforts to meet the needs of patients in relation to the replacement of lost teeth with dental implants considering the high success rate of osseointegration. ${ }^{1,2}$ Abutments are considered one of the most important prosthetic components of implant-supported restorations because they establish a relationship between the intraosseous structure of the implant and the implant-supported restoration. The transmucosal segment of abutments, which is adjacent to the connective tissue, and the peri-implant epithelium of the mucosa should be biocompatible so as not to cause inflammation in peri-implant tissues. ${ }^{3-5}$

It is very important to control inflammation around dental implants to decrease resorption of bone around the implant neck, help maintain the health of adjacent soft tissues, and increase the efficacy and longevity of implants. ${ }^{6}$ Several factors play a role in inducing inflammation around dental implants, including inadequate 
plaque control, absence of attached gingiva around the implant, the great thickness of gingiva around the implant neck, and the position of the margin of the implantsupported crown relative to the free gingival margin. ${ }^{6,7}$ A study reported that the primary etiologic factor for inflammation in peri-implant tissues is the infection due to anaerobic bacteria. Another etiologic agent for periimplant inflammation is the material type of the abutment used. ${ }^{8}$ The absence of irritation of cells around implants and abutments is a prerequisite for the long-term homeostasis in the peri-implant mucosa. ${ }^{3-5}$

Two abutments that have been recommended for the fabrication of implant-supported restorations are titanium and ceramic abutments. ${ }^{1}$ Ceramic abutments have some specific properties, including color matching, biocompatibility, and tissue compatibility, and they have now become very popular due to the extensiveness of ceramic systems, ${ }^{9}$ while titanium abutments result in a gray and unesthetic halo beyond the gingival margin of implants due to the reflection of light from the surface of titanium implants. The use of alumina and zirconia ceramics for the fabrication of abutments has become very popular, which is due to their color, light conduction properties, and high resistance against fracture. ${ }^{10,11}$

At present, the majority of implant systems present ceramic abutments, and some of these abutments are introduced in a prefabricated form in the system and some are customized. ${ }^{9,11}$ Despite all the favorable properties of ceramic abutments, they have some limitations too, including the fact that the ceramic-metal interface is prone to abrasion. The possible abrasion due to force at the implant-abutment interface between the zirconia abutment and the titanium external attachment might compromise the mechanical properties and adaptation between the implant and abutment, which might be an important factor for the transfer of tensions, causing possible mechanical problems in the implant prosthesis. Some studies have shown that all-ceramic abutments cannot be fabricated as precisely as the metal types; therefore, the resultant misfit might result in mechanical problems, such as screw loosening or biologic problems, such as bone loss due to accumulation of microorganisms. ${ }^{9,11}$

Adaptation between the external hexagon of the abutment and the internal hexagon of the implant should occur in a manner to allow a freedom of movement $<5 \mathrm{~mm}$ to stabilize the attachment between the abutment and implant. A lack of transverse and vertical adaptation transfers the forces to the abutment screw, implant, and bone, resulting in loosening and fracture or small fractures in bone, creating ischemic areas. ${ }^{10}$ The difference in biocompatibility of abutments on the one hand and stresses exerted on peri-implant tissues that result from a possible lack of complete adaptation between the abutment and fixture, on the contrary, might play a role in inducing proinflammatory cytokines. ${ }^{10}$

Cytokines are peptide mediators that have a role in the regulation of immunologic responses, local systemic inflammatory responses, and reparative responses in the face of invading agents. They exert their effect through induction of proliferation and differentiation of cells or prevention of cell proliferation and differentiation. ${ }^{12}$ Considering the transfer of tension and forces to peripheral tissues, biologic mechanisms and responses of the host tissues begin around the implants. The most important proinflammatory cytokines are interleukin (IL)-1 $\beta$ and IL-6 with similar and synergistic effects. Proinflammatory cytokines promote the synthesis of endothelium-binding molecules and inflammatory cells, such as neutrophils, monocytes, and fibroblasts and result in vasodilation, chemotaxis, and inflammation in the area. ${ }^{13,14}$

Nogueira-Filho et al ${ }^{15}$ compared the proinflammatory cytokine levels in the peri-implant GCF and in GCF of other oral areas and concluded that there was no significant difference in IL-4, IL-6, and IL-10 levels, and there were no changes over time, either. It should be pointed out that in the study above, the differences in abutment types were not evaluated.

In another study in 2015, the effect of different types of abutment (titanium, titanium nitride, and zirconia) on the peri-implant soft tissues was evaluated, and no significant differences were reported between different abutments in the variables evaluated around the implants after 2 years. ${ }^{16}$ All the variables evaluated in that study were clinical, and the conclusions were based on the evaluation of clinical parameters, including bleeding on probing and pocket depths.

Lops et $\mathrm{al}^{17}$ evaluated implants with zirconia and titanium abutments, and concluded that after 5 years of service of these implants in the oral cavity as single-unit implants, and in the posterior areas, there were no significant differences in radiographic and biologic parameters and even bone loss between these two implant types and even between them and natural teeth, consistent with the results of studies by Sailer et $\mathrm{al}^{18}$ and Zembic et al. ${ }^{19}$

In another study by Barwacz et $\mathrm{al}^{20}$ on proinflammatory markers around titanium and zirconia abutments, no significant differences were found between the two groups. It should be pointed out that this study was retrospective and the implant systems and the qualities of abutment implant attachments were not uniform in all the samples.

Based on what was discussed above and also a literature review, no prospective study is available with standardized samples in relation to comparison of proinflammatory cytokines between ceramic and titanium 
implants. On the contrary, the studies mentioned above have not evaluated the effect of confounding factors, such as implant location, the direction of the forces, and the position of the implant restoration margin in relation to the gingiva, which might be factors for induction of inflammation. Therefore, this study was undertaken to evaluate the proinflammatory factors around titanium and ceramic abutments by considering the factors mentioned above in a prospective clinical trial with an intraindividual design.

\section{MATERIALS AND METHODS}

The sample size was calculated at 15 samples in each group based on the results of a pilot study by considering a mean difference of 7.37 between the two abutment types with standard deviations of 4.59 and 6.48 and by considering $\alpha=0.05$ and a study power of $80 \%$. Allceramic crowns were fabricated for ceramic implants and metal-ceramic crowns were fabricated for titanium abutments. The protocol of the study was approved by the Ethics Committee of Tabriz University of Medical Sciences under the code IR.TBZMED.REC.1395.277.

The subjects were selected from the patients with posterior implants, referring to the dental offices of two prosthodontists for fabrication of prostheses based on inclusion and exclusion criteria.

\section{Inclusion Criteria}

The following inclusion criteria ${ }^{17}$ were considered: The presence of at least two posterior implants on two sides in the \#6 or \#7 areas in the same jaw, whose clinical success had been confirmed; the presence of at least $1 \mathrm{~mm}$ of attached gingiva around the implants; a maximum gingival thickness of $3 \mathrm{~mm}$ in the implant neck area; and a minimum age of 18 years.

\section{Exclusion Criteria}

The following exclusion criteria ${ }^{17}$ were considered: Subjects who had undergone scaling during the previous 3 months, pregnant and breastfeeding women, subjects who had received antibiotics during the previous 6 months, any systemic condition, such as diabetes affecting the oral health, drug and alcohol abuse and smoking, a history of the use of bisphosphonates, and use of systemic anti-inflammatory agents.

\section{Procedural Steps}

All the implant systems evaluated were Dentis, and the implant locations were in the \#6 and \#7 areas bilaterally. Abutments were selected with a uniform diameter of $55 \mathrm{~mm}$. The titanium abutments were prefabricated and ceramic abutments were customized by a pixdent machine (Iranian Bonyan Mechatronic Company, Iran). Computer-aided design-computer-aided manufacturing system was used to fabricate Cercon all-ceramic crowns in the laboratory in a uniform manner and by one single operator. All the crown margins were placed supragingivally.

All the subjects were instructed in effective plaque control around the healing abutment with a toothbrush, water jet, and chlorhexidine mouthwash for 2 weeks after placement of healing abutment screw (HAS). After 2 weeks, the subjects were recalled for the evaluation of plaque control based on the instructions. The design of this clinical trial was split mouth; in this context, titanium abutments were used on one side and ceramic abutments in the corresponding site on the other side.

After the laboratory procedures, in the crown delivery session, samples were taken from the GCF. After 4 months of prosthetic function, samples were taken again from the GCF. During this 4-month interval, the subjects were recalled for the control of oral hygiene.

To take samples from the GCF, after cleaning the oral cavity (half an hour after 3 minutes of thorough brushing), each area was isolated by cotton rolls and dried with a current of air for 5 seconds to remove all salivary contaminations. Sampling was carried out with the use of paper points (META BIOMED Co., Ltd., Chungbuk, Korea). The paper points were inserted into the gingival sulcus in four distinct areas (mesiobuccal, distobuccal, mesiolingual, and distolingual) until moderate resistance was observed. Then, each paper point was left in place for 30 seconds. Then, each paper point was placed in a sterile small tube and frozen at $-80^{\circ} \mathrm{C}$ until all the samples were collected and sent to the immunology laboratory for the determination of IL- 6 and IL-1 $\beta$ proinflammatory cytokine levels with the use of an enzyme-linked immunosorbent assay (ELISA) kit. It should be pointed out that any paper point with blood contamination was excluded from the study. ${ }^{17}$

\section{Immunology Laboratory Procedures}

All the laboratory procedures were carried out in the Immunology Laboratory of the Immunology Research Center of Tabriz University of Medical Sciences. On the test day, after defrosting the samples, $100 \mathrm{~mL}$ of phosphate-buffered saline was added to each sample and centrifuged at $3000 \mathrm{~g}$ at $4^{\circ} \mathrm{C}$ for 5 minutes. The resultant solutions were evaluated with the use of C-biosc ELISA kit to determine IL-1 $\beta$ and IL-6 concentrations using the ELISA sandwich technique as follows. Microplates covered with monoclonal antibodies against IL-6 and IL-1 $\beta$ were used. First, $50 \mathrm{~mL}$ of the sample solution and 
$50 \mathrm{~mL}$ of the special assay buffer were added to each well, and standard serial dilutions were prepared and added to the relevant well. Then, $50 \mathrm{~mL}$ of monoclonal anti-IL-6 and monoclonal anti-IL-1 $\beta$ conjugated with biotin were added to each well. After 2 hours of conjugation at room temperature on a rotator and 4 times of washing, $100 \mathrm{~mL}$ of streptavidin was added to each well and after 1 hour of incubation at room temperature, they were washed; then, $100 \mu \mathrm{L}$ of the tetramethylbenzidine (TMB) substrate was added to each well. After 15 minutes, the reaction was stopped with the use of stop solution and the contents of the plate were read with the use of an ELISA reader at a wavelength of $450 \mathrm{~nm}$. Then, standard graphs were drawn, and IL- 6 and IL-1 $\beta$ concentrations were determined on the graphs in $\mathrm{pg} /$ $\mathrm{mL}$. Each sample was tested several times to increase the accuracy of measurements and for quality control.

Data were analyzed with statistical indexes and techniques, including means, standard deviations, and multivariate regression analysis. Attempts were made to evaluate the concentrations of both cytokines individually in all the samples and as means in both groups.

\section{RESULTS}

In the present study, first, the normality of data was evaluated with Kolmogorov-Smirnov test. The result showed normal distribution of data ( $p>0.05)$.

Based on the results of the present study:

- The mean concentrations of IL-6 in the ceramic and titanium abutment groups were 25.11 and $46.17 \mathrm{pg} /$ $\mathrm{mL}$ respectively, indicating a significantly higher level of the cytokine in the titanium group $(\mathrm{p}<0.001$; Table 1 and Graph 1)

- The mean concentrations of IL-1 $\beta$ in the ceramic and titanium abutment groups were 13.05 and $20.42 \mathrm{pg} / \mathrm{mL}$

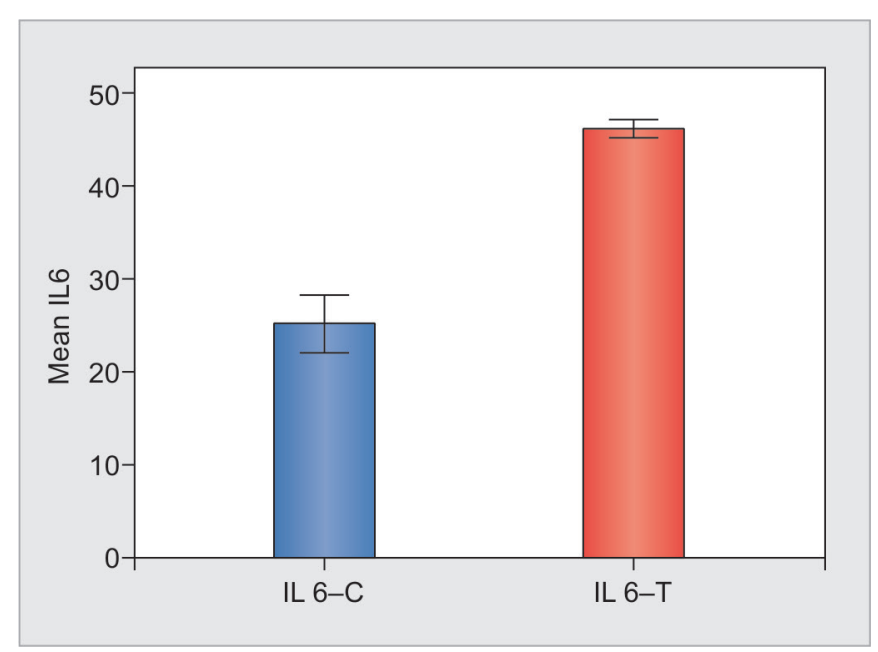

Graph 1: Comparison of IL-6 proinflammatory cytokine in the gingival crevicular fluid around implants with ceramic and titanium abutments
Table 1: The descriptive statistics of the study groups

\begin{tabular}{lllll}
\hline Study groups & $N$ & Min & Max & Mean \pm SD \\
\hline IL1 $\beta C$ & 8 & 6.40 & 22.34 & $13.05 \pm 4.59$ \\
IL1 $\beta T$ & 6 & 12.24 & 31.46 & $20.42 \pm 6.48$ \\
IL6C & 7 & 18.30 & 29.43 & $25.11 \pm 3.38$ \\
IL6T & 9 & 44.66 & 48.29 & $46.17 \pm 1.25$ \\
\hline
\end{tabular}

SD: Standard deviation

respectively, with no significant difference between the test groups based on the results of $t$-test $(p=0.098$; Table 1 and Graph 2)

- Comparison of the concentrations of IL-1 $\beta$ and IL-6 in the ceramic abutment group showed a higher concentration of IL- 6 than that of IL- $1 \beta$, which was significant based on the results of paired t-test $(\mathrm{p}<0.0001 ;$ Graph 3)

- Comparison of the concentrations of IL-1 $\beta$ and IL-6 in the titanium abutment groups showed that the concentration of IL- 6 was higher than that of IL-1 $\beta$, which was significant based on the results of paired t-test ( $\mathrm{p}<0.0001$; Graph 4)

- Comparison of the concentrations of IL- 6 and IL-1 $\beta$ showed higher concentrations of IL- 6 in titanium and ceramic abutments than those of IL-1 $\beta$, which was statistically significant ( $<<0.0001$; Graphs 3 and 4).

\section{DISCUSSION}

Titanium abutments have been recognized as the gold standard for implant-supported prosthetic restorations in all the jaw areas. One of the major disadvantages of these abutments is their gray color, which results in gray discoloration in the peri-implant mucosa. ${ }^{21,22}$ Zirconia abutments have been considered as an alternative in areas that are important from an esthetic viewpoint. The esthetic advantages of ceramic abutments over metallic type have been confirmed in recent clinical trials. ${ }^{23}$ One

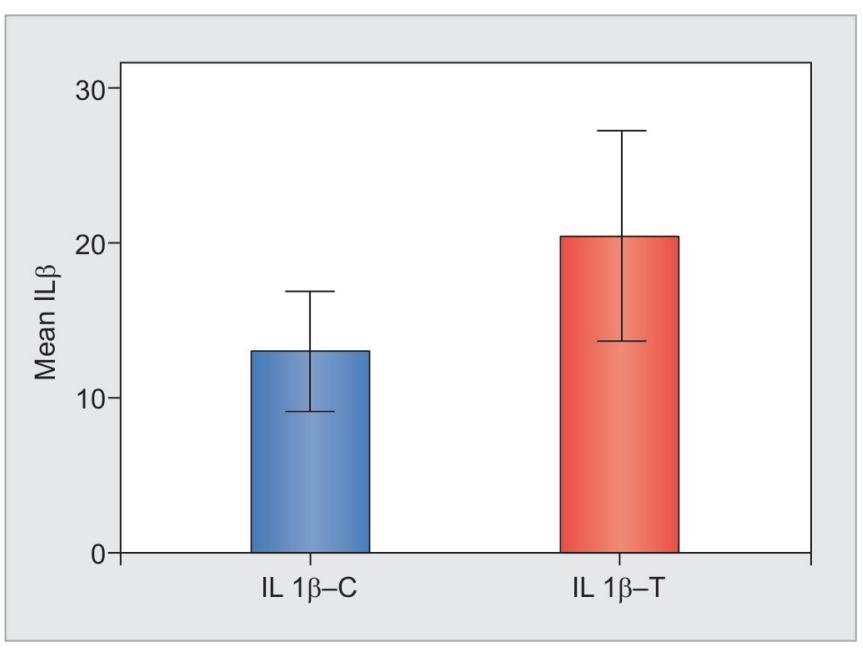

Graph 2: . Comparison of IL-1 $\beta$ proinflammatory cytokine in the gingival crevicular fluid around implants with ceramic and titanium abutments 


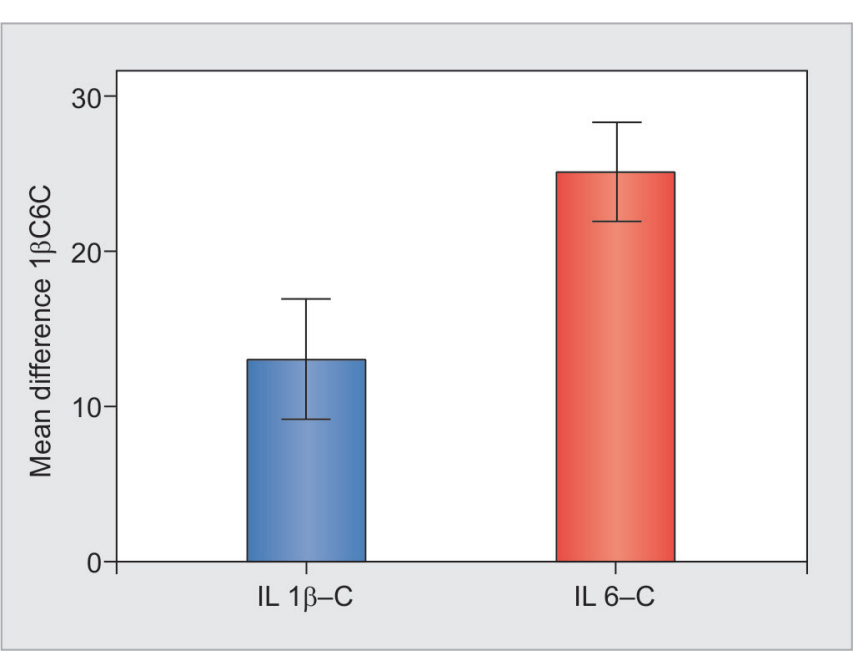

Graph 3: Comparison of concentrations of proinflammatory cytokines in the gingival crevicular fluid around implants with ceramic abutments

of the aspects in relation to the evaluation of zirconia abutments is their strength and resistance to fracture, which have been confirmed in various studies. ${ }^{24}$ A study on the biologic and radiographic parameters of titanium and zirconia abutments for single-unit implant-supported prostheses did not reveal any fractures in implants after 5 years of function in the posterior areas, and all the implants exhibited normal function up to 5 years. In addition, there were no significant differences in the biologic and radiographic parameters evaluated between the two implant types. Based on the results of that study, the medium-term longevity of zirconia abutments was similar to that of titanium abutments; however, the researchers suggested that further studies are necessary to confirm it in the long term. ${ }^{17}$ It should be pointed out that in that study, too, similar to the present study, a twostage protocol was used for the evaluation of abutments.

In another randomized controlled clinical trial, too, zirconia and titanium abutments in single-unit and posterior implants were evaluated. In that study, 40 implants were evaluated in 22 subjects; 20 implants were zirconia and 20 implants were titanium. The results of the study showed no treatment failure in the subjects. In addition, the biologic, esthetic, and technical findings were similar in both abutments after 3 years. ${ }^{19}$ In that study, too, only periodontal parameters were evaluated.

Another factor, which is one of the most effective factors influencing the success or failure of implant treatments, is the biocompatibility of abutments. In other words, this factor is the absence of irritation of periodontal tissues and bone by the implant, which is important for confirmation of the use of abutments in the clinic. Determination of proinflammatory cytokines is one of the reliable methods for the evaluation of this property.

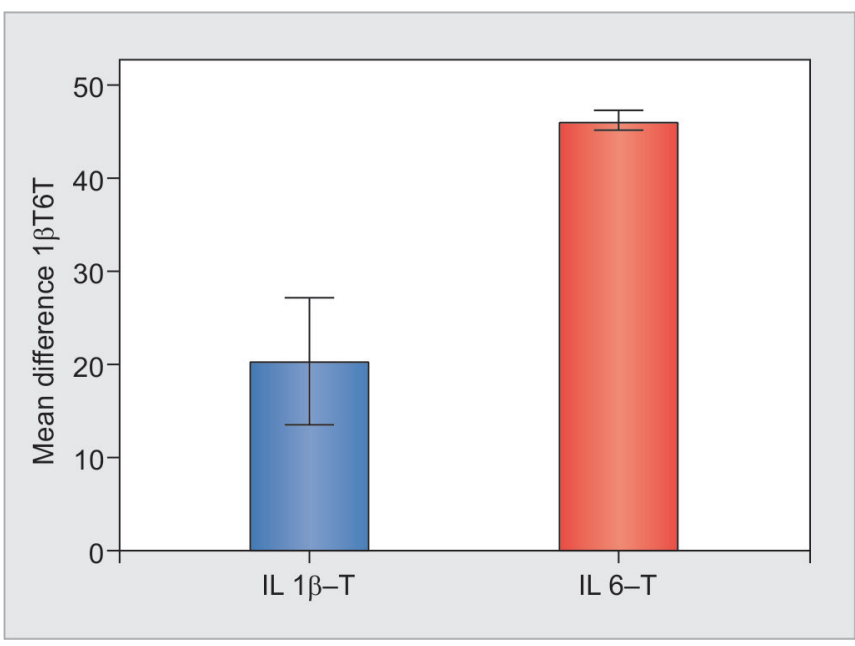

Graph 4: Comparison of concentrations of proinflammatory cytokines in the gingival crevicular fluid around implants with titanium abutments

In the present clinical trial, the concentrations of IL-1 $\beta$ and IL-6 proinflammatory cytokines in the GCF around two commonly used abutments were evaluated. The results showed that the mean concentrations of IL-1 $\beta$ and IL-6 in ceramic abutments were lower than those in the titanium abutments. In addition, statistical comparisons between IL- 6 and IL- $1 \beta$ showed that the concentration of IL- 6 was higher than that of IL- $1 \beta$ in titanium and ceramic abutments.

A study compared the proinflammatory cytokines and mediators involved in bone metabolism around zirconia and titanium abutments after at least 6 months of clinical function and showed that the abutment material type and patient gender and age had no effect on the expression of the majority of mediators and proinflammatory cytokines. The molecular findings of GCF around implants, too, showed that both abutments were clinically biocompatible. ${ }^{20}$ It should be pointed out that in that study of the cytokines evaluated, similar to that in the present study, the concentrations of IL- $1 \beta$ and IL- 6 around titanium implants were significantly higher than those around ceramic abutments. In another study, too, long-term comparison of cytokines in the fluids and GCF around implants showed that the concentrations of IL-6, IL-4, IL-10, tumor necrosis factor- $\alpha$, and interferon- $\delta$ in the GCF around implants and teeth were not significantly different, with no significant changes over time. The general conclusion of that study was that the human body immune response was the same in peri-implant tissue and in periodontal tissues after evaluation of cytokines. ${ }^{15}$ The results of that study do not coincide with the results of the present study, and it should be pointed out that the abutment material types were not reported in that study; a one-stage protocol was used and only one cytokine was evaluated. 


\section{CONCLUSION}

The results of this study showed that the mean concentrations of IL- $1 \beta$ and IL- 6 in the ceramic abutment group were lower than those in the titanium abutment group, and the difference was significant in the case of IL-6 only. In addition, comparison of the mean concentrations between IL- 6 and IL-1 $\beta$ showed that the concentrations of IL-6 in titanium and ceramic abutments were higher than those of IL-1 $\beta$.

\section{REFERENCES}

1. Foley $\mathrm{CH}$, Kerns DG, Hallmon WW, Rivera-Hidalgo F, Nelson CJ, Spears R, Spears R, Dechow PC, Opperman LA. Effect of phosphate treatment of acid-etched implants on mineral apposition rates near implants in a dog model. Int J Oral Maxillofac Implants 2010 Mar-Apr;25(2):278-286.

2. Schropp L, Isidor F. Timing of implant placement relative to tooth extraction. J Oral Rehabil 2008 Jan;35 (Suppl 1):33-43.

3. Norton MR. Understanding the intimate relationship between biomechanics and optimal clinical performance: application of implant design. Compend Contin Educ Dent 2002 Sep;23 (9 Suppl 2):21-25.

4. Ratner BD. Replacing and renewing: synthetic materials, biomimetics, and tissue engineering in implant dentistry. J Dent Educ 2001 Dec;65(12):1340-1347.

5. Steigenga JT, Al-Shammari KF, Nociti FH, Misch CE, Wang HL. Dental implant design and its relationship to longterm implant success. Implant Dent 2003 Feb;12(4):306-317.

6. Berglundh T, Lindhe J, Ericsson I, Marinello CP, Liljenberg B, Thomsen P. The soft tissue barrier at implants and teeth. Clin Oral Implants Res 1991 Apr-Jun;2(2):81-90.

7. Kuppusamy M, Watanabe H, Kasugai S, Kuroda S. Effects of abutment removal and reconnection on inflammatory cytokine production around dental implants. Implant Dent 2015 Dec;24(6):730-734.

8. Mombelli A, Lang NP. The diagnosis and treatment of Periimplantitis. Periodontol 20001998 Jun;17:63-76.

9. Silverstein L, Garg A, Callan D, Shatz P. The key to success: maintaining the long-term health of implants. Dent Today 1998 Feb;17(2):104, 106, 108-111.

10. Bauman GR, Mills M, Rapley JW, Hallmon WW. Implant maintenance: debridement and peri-implant home care. Compendium 1991 Sep;12(9):644, 646, 648.

11. Humphrey S. Implant maintenance. Dent Clin North Am 2006 Jul;50(3):463-478.

12. Springstead $M$, Thomas $M$, Cline N. Educating a patient with dental implants. Dent Assist 1992;62(2):5-8.
13. Hillenburg K, Kosinski T, Mentag P. Control of Periimplant inflammation. Pract Peridontics Aesthet Dent 1990 Nov-Dec;3(8):11-16.

14. Kracher CM, Smith WS. Oral health maintenance dental implants. Dent Assist 2010 Mar-Apr;79(2):27-35.

15. Nogueira-Filho G, Pesun I, Isaak-Ploegman C, Wijegunasinghe M, Wierzbicki T, McCulloch CA. Longitudinal comparison of cytokines in peri-implant fluid and gingival crevicular fluid in healthy mouths. J Periodontol 2014 Nov;85(11): 1582-1588.

16. Ferrari M, Cagidiaco MC, Franklin GG. Effect of different prosthetic abutments on peri-implant soft tissue. A randomized controlled clinical trial. Am J Dent 2015 Apr;28(2):573-576.

17. Lops D, Bressan E, Chiapasco M, Rossi A, Romeo E. Zirconia and titanium implant abutments for single-tooth implant prostheses after 5 years of function in posterior regions. Int J Oral Maxillofac Implants 2013 Jan-Feb;28(1):281-287.

18. Sailer I, Zembic A, Jung RE, Siegenthaler D, Holderegger C, Hämmerle $\mathrm{CH}$. Randomized controlled clinical trial of customized zirconia and titanium implant abutments for canine and posterior single-tooth implant reconstructions: preliminary results at 1 year of function. Clin Oral Implants Res 2009 Mar;20(3):219-225.

19. Zembic A, Sailer I, Jung RE, Hämmerle CH. Randomizedcontrolled clinical trial of customized zirconia and titanium implant abutments for single-tooth implants in canine and posterior regions: 3-year results. Clin Oral Implants Res 2009 Aug;20(8):802-808.

20. Barwacz CA, Brogden KA, Stanford CM, Dawson DV, Recker EN, Blanchette D. Comparison of pro-inflammatory cytokines and bone metabolism mediators around titanium and zirconia dental implant abutments following a minimum of 6 months of clinical function. Clin Oral Implants Res 2015 Apr;26(4):e35-e41.

21. Park SE, Da Silva JD, Weber HP, Ishikawa-Nagai S. Optical phenomenon of peri-implant soft tissue. Part I. Spectrophotometric assessment of natural tooth gingiva and peri-implant mucosa. Clin Oral Implants Res 2007 Oct;18(5):569-574.

22. Jung RE, Sailer I, Hämmerle $\mathrm{CH}$, Attin T, Schmidlin P. In vitro color changes of soft tissues caused by restorative materials. Int J Periodontics Restorative Dent 2007 Jun;27(3):251-257.

23. Jung RE, Holderegger C, Sailer I, Khraisat A, Suter A, Hämmerle $\mathrm{CH}$. The effect of all-ceramic and porcelain-fusedto-metal restorations on marginal Peri-implant soft tissue color: a randomized controlled clinical trial. Int J Periodontics Restorative Dent 2008 Aug;28(4):357-365.

24. Canullo L. Clinical outcome study of customized zirconia abutments for single-implant restorations. Int J Prosthodont 2007 Sep-Oct;20(5):489-493. 